\title{
Ketamine for depression relapse prevention following electroconvulsive therapy: protocol for a randomised pilot trial (the KEEP-WELL trial)
}

Martha Finnegan ${ }^{1}$, Karen Ryan ${ }^{2}$, Enda Shanahan ${ }^{1}$, Andrew Harkin ${ }^{3}$, Leslie Daly ${ }^{4}$ and Declan M. McLoughlin ${ }^{1 *}$

\begin{abstract}
Background: Major depressive disorder is a common debilitating illness that is the second leading contributor to the global burden of disease. Unfortunately, about $30 \%$ of patients do not respond to adequate trials of antidepressants and/or psychotherapies. About 45-60 \% of such treatment-resistant patients will remit with electroconvulsive therapy (ECT). However, relapse rates are high following ECT_-38\% after 6 months. There is a need for better relapse prevention strategies. One possibility is to use ketamine, a competitive glutamate receptor antagonist used for anaesthesia. A recent paradigm shift in treating depression and understanding its biology has been the finding that ketamine has a robust, rapid-onset, though short-lived, antidepressant effect that is possibly mediated through neuroplastic effects. However, ketamine has not previously been reported on for relapse prevention.
\end{abstract}

Methods/design: The main objective of this study is to conduct a randomised controlled pilot trial $(n=40)$ of a 4-week course of once-weekly ketamine infusions for relapse prevention following ECT for depression to assess trial procedures that will inform a future definitive trial. Participants with unipolar depression will be recruited prior to commencing ECT and be assessed weekly during the ECT course using the primary clinical outcome, the 24-item Hamilton Rating Scale for Depression (HRSD-24). Those who meet standard response criteria will be invited, on completing ECT, to be randomised in a 1:1 ratio to a course of four once-weekly infusions of ketamine or an active comparator midazolam, which mimics some of the effects of ketamine and may improve blinding over inactive placebo. Participants will be followed up over 6 months using the HRSD-24 to assess for relapse.

Discussion: This is the first registered trial (NCT02414932, https://clinicaltrials.gov/ct2/show/NCT02414932) of ketamine for depression relapse prevention, an important possible use of this agent. The primary focus of the pilot trial is on feasibility. However, a $95 \%$ confidence interval will be determined for the difference between ketamine and midazolam groups in 6-month relapse rates to help inform a future definitive trial.

Trial registration: https://clinicaltrials.gov/ NCT02414932

Secondary Identifying numbers:

EudraCT number: 2014-000339-18

Sponsors' Reference, Sponsor: St. Patrick's Mental Health Services: 05/14

Research Ethics Committee Reference, Joint REC of St James' and Tallaght Hospitals, Dublin: 2014-08-19

Keywords: Depression, Relapse prevention, Ketamine, Electroconvulsive therapy, Pilot trial

\footnotetext{
*Correspondence: d.mcloughlin@tcd.ie

'Department of Psychiatry and Trinity College Institute of Neuroscience, St.

Patrick's University Hospital, James' St., Dublin 8, Ireland

Full list of author information is available at the end of the article
} 


\section{Background}

\section{Depression, ECT and relapse}

Major depressive disorder (MDD) is a debilitating mental illness with a lifetime prevalence of $12-20 \%$ [1]. It is the most costly brain disorder in Europe, accounting for $1 \%$ ( $€ 118$ billion annually) of the total European economy [2]. Indeed, depression is currently the second largest cause globally for years lived with disability [3].

About $30 \%$ of patients do not respond to antidepressants even after multiple trials with/without psychotherapies [4]. However, electroconvulsive therapy (ECT) offers up to $60 \%$ of such treatment-resistant patients to complete remission [5-7]. ECT is a medically safe procedure and is more acutely effective than psychotherapy or antidepressants for severe, often treatment-resistant, depression [5]. The major concerns are cognitive side effects, but for most people, these are transient and many cognitive functions improve [8]. Treatments involve passing small electrical charges through the brain to induce a seizure lasting $\sim 30 \mathrm{~s}$ under anaesthesia with a muscle relaxant. Six to 12 treatments are typically administered in a course, two to three times weekly [9]. Worldwide, 1.4 million people receive ECT annually, including nearly 260 people in Ireland [10].

In a recent meta-analysis, we found that taking antidepressants following successful ECT halves the risk for relapse (risk ratio $=0.49, p<0.0001$, NNT $=3.3$ ) at 6 months from nearly $80 \%$ [11], but mean relapse rates remain high: $27.1 \%$ after 3 months and $37.7 \%$ after 6 months [11]. Even continuation ECT (C-ECT), albeit mostly at nonadjustable fixed schedules, does not seem to improve 6 -month relapse rates $(37.2 \%)$. Notably, these relapse rates are similar to those for patients who respond only after $\geq 3$ antidepressant steps and most likely reflect the recurrent nature of treatment-resistant depression [4].

A major challenge now is how best to prevent relapse after successful treatment of depression with ECT. However, remarkably, the evidence base for relapse prevention in depression following any successful treatment is small. For example, the National Institute for Health and Care Excellence (NICE) in the UK have identified that evidence on relapse prevention in depression is limited and recommended research in this area. [12] To date, the reported randomised controlled trials for relapse prevention following successful antidepressant therapy have focused on the effect of 12 months' tricyclic or SSRI antidepressant therapy, showing consistent but limited reduction in relapse rates [13]. As discussed in our review [11], there have been very few randomised, controlled trials focusing on relapse prevention after ECT. One study investigated the use of nortriptyline or nortriptyline and lithium for relapse prevention following successful ECT for depression [14]. Nortriptyline-lithium combination therapy had a marked advantage in time to relapse, superior to both placebo and nortriptyline alone. Other studies have focused on fixed-schedule continuation ECT, which has a relapse prevention effect in wellchosen groups [15].

\section{Ketamine as an antidepressant}

Ketamine is a competitive glutamate $N$-methyl-D-aspartate receptor (NMDAR) antagonist with a half-life of 2-3 h. Ketamine has a remarkably rapid antidepressant effect, targeting core symptoms, in treatment-resistant depression when given as single sub-anaesthetic doses, usually a $40-\mathrm{min} 0.5 \mathrm{mg} / \mathrm{kg}$ intravenous infusion [14]. Thereafter, robust antidepressant effects $(\sim 70 \%$ responder rates) occur within 2-4 h and persist for a few days, i.e. beyond immediate NMDAR blockade [14]. These findings have led to the most exciting development in treating and understanding depression in over 50 years and represent a paradigm shift away from conventional slow-acting monaminergic antidepressants. Preclinical studies have shown that within just $2 \mathrm{~h}$, ketamine increases synaptogenesis and spine formation in rodent prefrontal cortex and rapidly reverses chronic stress-induced depressive behaviours and prefrontal neuronal atrophy [15]. These effects are mediated, at least in part, via Akt/GSK-3/mammalian target of rapamycin (mTOR) signalling and increased dendritic translation of synaptic proteins [16], as well as deactivation of eukaryotic elongation factor 2 (eEF2) kinase, resulting in de-suppression of brain-derived neurotrophic factor (BDNF) translation [17]. BDNF mediates synaptic plasticity and is implicated in mechanisms of antidepressants and ECT [18]. Changes in blood mononuclear cell levels of phosphorylated mTOR, eEF2 and GSK-3beta have also been associated with response to ketamine [19], suggesting potential as biomarkers for response.

Ketamine is psychotomimetic, but at low dosage, it is safe, with patients and healthy controls occasionally experiencing mild dissociative and psychotic symptoms that resolve soon after finishing infusions [16-18]. To control for these effects, and also avoid "carry-over" effects in crossover studies while improving blinding, midazolam, at the sub-anaesthetic dose of $0.045 \mathrm{mg} / \mathrm{kg}$, has been used as a control in parallel-group design trials rather than inactive placebo saline [19]. Ketamine can be a drug of abuse and chronic high-dose abuse can cause uropathy and dependency. However, repeated (e.g. 2-3/ week for 2 weeks) infusions of sub-anaesthetic ketamine are safe with more sustained antidepressant effects [20, 21]. Two recent reviews of trials of ketamine for use as an antidepressant showed the most commonly used dosage is a 40 -min infusion of $0.5 \mathrm{mg} / \mathrm{kg}[16,22]$. Bioavailability of ketamine is highest when administered intravenously [23]. In sub-anaesthetic doses, ketamine is a medically safe drug but can cause transient rises in 
pulse and blood pressure during infusion and for up to 80 min afterward. However, a recent review of ketamine in depression concluded that outside recreational usage, there have been no reports of persistent adverse effects with sub-anaesthetic uses of ketamine [24].

The effect of ketamine on cognition is unclear and has only been studied in acute treatment of depression [25]. There may be changes in visual and working memory [26] and an association between baseline neurocognitive performance and response to ketamine [27]. Optimum dosing and deliver of ketamine has not been established [25]. Ketamine has been used for ECT anaesthesia and is associated with earlier improvement and possibly fewer cognitive side effects but no overall better response [16, $20,22,28]$. There have been insufficient studies of intramuscular, oral or intranasal ketamine for depression to currently warrant studying these preparations for relapse prevention [21]. While the half-life of ketamine is $3 \mathrm{~h}$, in previous studies, the antidepressant effect was maintained for up to 2 weeks [29]. No trials have yet been reported, or registered, for using ketamine as an adjunctive treatment to reduce relapse rates following successful depression treatment-a potential use of ketamine that this trial will explore.

\section{Methods/design}

\section{Study objective}

The primary objective is to conduct a randomised, controlled, patient- and rater-blinded pilot study of ketamine vs. an active comparator (midazolam) for 4 weeks following successful ECT, to assess trial process to inform a future definitive trial.

\section{Secondary objective}

To calculate a $95 \%$ confidence interval for an unadjusted hazard ratio that will allow interpretation of statistical difference between ketamine and midazolam groups to assess ketamine for reducing 6-month relapse rates following successful ECT.

\section{Overview}

This randomised, controlled pilot trial will take place over 30 months. The study will have an open recruitment phase (phase I) followed by a randomised treatment phase (phase II). We will initially recruit patients with unipolar major depressive disorder (Diagnostic and Statistical Manual of Mental Disorders, Fourth Edition (DSM-IV) criteria [30]) referred for ECT, who will be assessed weekly to identify those eligible to take part in the randomised controlled pilot trial in phase II. Participants who are successfully treated with ECT (phase 1) and continue to meet inclusion criteria will be randomly allocated in a 1:1 ratio to a 4-week course of either once-weekly ketamine at $0.5 \mathrm{mg} / \mathrm{kg}$ or the active comparator midazolam at $0.045 \mathrm{mg} / \mathrm{kg}$ (phase 2). The trial will take place under "real world" conditions with both groups continuing usual care (e.g. regular medications, psychological and other therapies and out-patient review) during the randomised treatment phase and thereafter. Participants will be followed up over 6 months following ECT to identify if and when relapse occurs.

\section{Site}

This single-site study will take place in St. Patrick's University Hospital, Dublin, an independent-sector 250bed university teaching hospital that provides a national mental health service. About one third of all ECT in Ireland is administered at the centre [7, 31].

\section{Research ethics approval}

Approval for this pilot trial was obtained from the joint authorised Research Ethics Committee of St. James' and Tallaght Hospitals, Dublin. Site approval was also obtained from the relevant committee at St. Patrick's University Hospital. Authorisation for the clinical trial was obtained from the Health Products Regulatory Authority of Ireland, the relevant body under the European framework for clinical trials, EudraCT (2014-000339-18). The study will be conducted in accordance with the principles that have their origin in the Declaration of Helsinki [32], in accordance with Good Clinical Practice (GCP), as defined by the International Conference on Harmonisation [33] $(\mathrm{ICH})$, and in accordance with the ethical principles underlying European Union Directive 2001/20/EC and 2005/28/EC. The trial has been registered at clinicaltrials.gov (NCT02414932).

\section{Recruitment}

In line with recommendations for pilot studies [34], a formal sample size calculation has not been performed. Twenty participants is an acceptable total number for the purposes of a pilot trial. For this pilot trial, we aim to recruit up to 20 patients per group, a total of 40. Response rates to ECT are $40-60 \%$ [5], so at least 66 patients need to be initially recruited. Allowing for a $15 \%$ drop-out rate, we will therefore seek to recruit 78 patients. We expect to recruit 78 participants within 16 months, 47 of whom will meet response criteria following ECT [5], and that 40 of these will additionally consent to be randomised and participate in the pilot trial.

\section{Consent}

Written informed consent will be obtained by members of the research team using the study-specific consent form (Additional file 1). Potential participants will be provided with an information leaflet and letter of invitation (Additional file 1) and verbal information at the first point of contact with a member of the research team. 


\section{Eligibility criteria}

Participants will be current inpatients in university teaching hospitals in St. Patrick's Mental Health Services, who have a diagnosis of unipolar MDD and are referred for ECT. Participants may be male or female, aged $\geq 18$ years, and from a variety of geographical (within Ireland) and socioeconomic backgrounds. Participants will not have any medical condition that would preclude treatment with ECT or ketamine/midazolam.

To be eligible for inclusion in phase 1, each participant must meet each of the following criteria at screening and must continue to fulfil these criteria at baseline.

1. Subjects must be able and willing to give written informed consent and comply with the requirements of this study protocol.

2. Diagnosed with unipolar major depressive disorder (DSM-IV), have a 24-item Hamilton Rating Scale for Depression (HRSD-24) of $\geq 21$ and be referred for ECT.

3. Female subjects of child-bearing potential and male subjects whose partner is of child-bearing potential must be willing to ensure that they or their partner use effective contraception during the randomised treatment phase (phase II) and for 5 weeks thereafter.

Subjects are excluded from the study if any of the following criteria are met at screening:

1. Allergy/sensitivity to study medications or their ingredients.

2. Subjects who have participated in another study and received any other investigational agent within 6 months.

3. Any condition rendering patient medically unfit for ECT; general anaesthesia; ketamine or midazolam-assessed by physical examination, routine haematology and biochemistry investigations prior to enrolment.

4. Medications that may significantly alter the pharmacokinetics of ketamine (e.g. ketoconazole, clarithromycin) are contraindicated during the trial, and participants taking any of these medications at screening will be excluded from the trial.

5. Subjects who have a history of drug or alcohol use that, in the opinion of the investigator, would interfere with adherence to study requirements.

6. Known history of, or documented positive hepatitis $\mathrm{B}$ or $\mathrm{C}$ or HIV infection, advanced malignancy or terminal illness.

7. Scheduled for non-trial procedures requiring general anaesthesia during the study.

8. Active suicidal intention.
9. Dementia, intellectual disability or a score on the standardised Mini Mental State Examination (sMMSE) of $<24$.

10. Lifetime history of bipolar affective disorder.

11. Current history of post-traumatic stress disorder.

12. Other axis I diagnosis (DSM-IV).

13. ECT in the 6 months prior to recruitment.

14. Currently a prisoner or residing in a nursing home.

For inclusion in the randomised controlled trial (phase 2), following successful ECT, patients must additionally have:

1. Received a significant course of ECT (i.e. at least five sessions)

2. Achieved at least response criteria (i.e. $\geq 60 \%$ decrease from baseline HRSD-24 score and score $\leq 16$ on two consecutive weekly ratings)

3. Have a nominated adult who can stay with them for 24-h on out-patient treatment days

4. sMMSE [35] score of $\geq 24$

\section{Assessments}

The primary clinical outcome measure is the relapse rate at 6 months as measured using the objectively rated HRSD-24 [36]. To enter the study, patients must score $\geq 21$. Subjective mood ratings will be also measured using the Quick Inventory of Depressive Symptoms, self-report version (QIDS-SR) [37]. Baseline assessment will also include diagnosis and treatment history: diagnosis of major depressive disorder will be confirmed using the mood episodes module of the Structured Clinical Interview for DSM-IV Axis I Disorders (SCID) [38]. The Maudsley Staging Method for Treatment Resistant Depression (MSTRD) [39] will be used to provide a measure of treatment-resistance. Handedness will be recorded with the Edinburgh Handedness Questionnaire (EHQ) [40]. The National Adult Reading Test (NART) [41] will measure premorbid intelligence.

Additional baseline data from patient interview and case-note review will include age, sex, weight, height, occupation, educational attainment, duration of index depressive episode, number of previous depressive episodes, previous ECT, history of medical illness and surgical treatments, personal and family history of alcohol/substance dependency, presence of psychotic symptoms (detected by SCID) and current medications and other therapies. Changes in medications will be documented at follow-up interviews (Table 1, schedule of events).

Participants will be assessed weekly during ECT using the HRSD-24 and QIDS-SR. Response to ECT is defined as achieving $\geq 60 \%$ decrease from baseline HRSD-24 and score $\leq 16$ on two consecutive weekly ratings. Remission criteria are $\geq 60 \%$ decrease in HRSD from baseline 
Table 1 Schedule of enrolment, assessments and interventions

\begin{tabular}{|c|c|c|c|c|c|c|c|}
\hline \multirow[b]{2}{*}{ Assessment } & \multicolumn{3}{|c|}{ Phase 1: ECT patients and healthy controls } & \multicolumn{4}{|c|}{ Phase 2: ECT responders randomised to ketamine or midazolam } \\
\hline & $\begin{array}{l}\text { Baseline } \\
\text { (pre-ECT) }\end{array}$ & $\begin{array}{l}\text { Weekly during } \\
\text { ECT course }\end{array}$ & $\begin{array}{l}\text { End of ECT } \\
\text { course }\end{array}$ & Pre-infusions & $\begin{array}{l}\text { Infusions 1-4; } \\
\text { weeks 1-4 }\end{array}$ & $\begin{array}{l}\text { Follow-ups: weeks } \\
6,8,12 \text { and } 20\end{array}$ & $\begin{array}{l}\text { Final follow-up } \\
\text { week } 26\end{array}$ \\
\hline \multicolumn{8}{|l|}{ Diagnosis and treatment } \\
\hline Background, SCID, NART, CTQ & $\checkmark$ & & & & & & \\
\hline Treatment review & $\checkmark$ & & $\checkmark$ & & $\checkmark(1-4)$ & $\checkmark(6-20)$ & $\checkmark$ \\
\hline \multicolumn{8}{|l|}{ Clinical outcomes } \\
\hline HDRS-24 & $\checkmark$ & $\checkmark$ & $\checkmark$ & & $\checkmark \checkmark \checkmark \checkmark(1-4)$ & $\checkmark(6-20)$ & $\checkmark$ \\
\hline QIDS-SR & $\checkmark$ & $\checkmark$ & $\checkmark$ & & $\checkmark \checkmark \checkmark \checkmark(1-4)$ & $\checkmark(6-20)$ & $\checkmark$ \\
\hline \multicolumn{8}{|l|}{ Cognitive outcomes } \\
\hline ACE-R & $\checkmark$ & & $\checkmark$ & & $\checkmark 4$ th & & $\checkmark$ \\
\hline Digit spans & $\checkmark$ & & $\checkmark$ & & $\sqrt{ } 4$ th & & $\checkmark$ \\
\hline Trails A + B & $\checkmark$ & & $\checkmark$ & & $\sqrt{ } 4$ th & & $\checkmark$ \\
\hline sMMSE & $\checkmark$ & & $\checkmark$ & & $\sqrt{ } 4$ th & & $\checkmark$ \\
\hline AMI & $\checkmark$ & & $\checkmark$ & & $\sqrt{ } 4$ th & & $\checkmark$ \\
\hline \multicolumn{8}{|l|}{ Ketamine effects } \\
\hline CADSS & & & & & $\checkmark \checkmark \checkmark \checkmark(1-4)$ & & \\
\hline BPRS & & & & & $\checkmark \checkmark \checkmark \checkmark(1-4)$ & & \\
\hline YMRS & & & & & $\checkmark \checkmark \checkmark \checkmark(1-4)$ & & \\
\hline PRISE & & & & & $\checkmark \checkmark \checkmark \checkmark(1-4)$ & & \\
\hline \multicolumn{8}{|l|}{ Consent } \\
\hline Signed consent & $\checkmark$ & & & $\checkmark$ & & & \\
\hline Verbal assent & $\checkmark$ & $\checkmark$ & $\checkmark$ & & $\checkmark \checkmark \checkmark \checkmark(1-4)$ & $\checkmark(6-20)$ & $\checkmark$ \\
\hline \multicolumn{8}{|l|}{ Eligibility } \\
\hline Eligibility check & $\checkmark$ & $\checkmark$ & $\checkmark$ & $\checkmark$ & $\checkmark \checkmark \checkmark \checkmark(1-4)$ & $\checkmark(6-20)$ & $\checkmark$ \\
\hline \multicolumn{8}{|l|}{ Randomisation } \\
\hline Allocation & & & & $\checkmark$ & & & \\
\hline
\end{tabular}

and score $\leq 10$ on two consecutive weekly ratings. Those identified as being ECT responders will be invited to participate in the two-group parallel-design randomised controlled pilot trial. The advantages of randomisation and blinding in this group will also be used to perform studies of peripheral blood biomarkers as potential predictors of response to ketamine (Additional file 2). During infusion sessions in the randomised treatment phase, HRSD-24 scores will be obtained $60 \mathrm{~min}$ before the infusion begins and at +120 and +240 min afterwards. Baseline scores on sleep and appetite items will be maintained for repeated measures within 1 day. The +240 min HRSD24 scores will serve as the weekly post-ECT scores up to follow-up week 4.

Ketamine psychotomimetic effects and adverse events will be assessed using the following instruments before, during (+35-40 min) and after (+240 min) infusions of ketamine or midazolam:

- Clinician-Administered Dissociative States Scale (CADSS) [42]
- Brief Psychiatric Rating Scale (BPRS; four-item positive symptom subscale) [43]

- Young Mania Rating Scale (YMRS; mood item) [44]

- Patient-Rated Inventory of Side Effects (PRISE) [45]

Participants will be followed up for 6 months following ECT with repeated questionnaires comprising treatment review plus HRSD-24 and QIDS-SR at weeks 6, 8, 12 and 20 post-ECT. Criteria for relapse are $\geq 10$ point increase in HRSD-24 compared to baseline phase 2 score plus HRSD $\geq 16$; in addition, increase in the HRSD should be maintained 1 week later (if indicated, additional follow-ups will be arranged). Hospital admission, further ECT and deliberate self-harm/suicide also constitute relapse. Timing of these events will be recorded. A final follow-up session in week 26 will comprise HRSD24, QIDS-SR and cognitive outcomes.

There are no published data on effects of ketamine on cognition in ECT responders. We will use the following battery pre- and post-ECT course, after the fourth infusion and at 6 -month follow-up. The post-ECT assessment will 
serve as baseline for the randomised pilot trial. Where appropriate, parallel versions will be used to reduce practice effects. Global cognition will be assessed with the sMMSE [35]. Immediate short-term memory, attention and working memory will be measured using Forward and Backward Digit Spans [46]. Motor and psychomotor speed will be assessed using the Trail Making Test (part A) [46]. Frontal-executive function will be rated by Trail Making Test (part B) [46] plus letter and category verbal fluencies [47]. Anterograde verbal memory will be tested using the verbal learning component of the Addenbrooke's Cognitive Examination III (delayed and immediate recall of a seven-item address) [48]. Retrograde amnesia for autobiographical information will be measured using the Kopelman Autobiographical Memory Interview (K-AMI) [49].

\section{Interventions}

Participants in phase I will receive ECT and usual care and will be monitored weekly using the HRSD-24 for response. ECT will be administered twice-weekly with hand-held electrodes according to Royal College of Psychiatrists' guidelines and as previously described [7, 50]. Briefly, the Mecta $5000 \mathrm{M}$ device (Mecta Corporation, USA) will be used and seizure duration measured by EEG monitoring. Methohexitone $(0.75-1.0 \mathrm{mg} / \mathrm{kg})$ will be used for anaesthesia with suxamethonium (0.5$1.0 \mathrm{mg} / \mathrm{kg})$ as muscle relaxant. Brief-pulse (1.0-ms pulse width; current amplitude $800 \mathrm{~mA}$ ) ECT will be administered twice weekly (Mecta $5000 \mathrm{M}$ device, Mecta Corp., Portland, Ore.; maximum $1200 \mathrm{mC}$ ), using methohexitone $(0.75-1.0 \mathrm{mg} / \mathrm{kg})$ anaesthesia and succinylcholine $(0.5-1.0 \mathrm{mg} / \mathrm{kg})$ for muscle relaxation $(16,22)$. Seizure threshold (ST) will be established by a method of limits, as previously described [7], at the first session, and subsequent treatments will be given at $1.5 \times \mathrm{ST}$ for BL ECT and $6.0 \times \mathrm{ST}$ for RUL ECT. Stimulus charge will be titrated upward as required during treatment courses following a standard stimulus dosing protocol. To reflect routine clinical practice, number of ECT treatments will be determined by referring physicians who will be blind to randomisation. ECT characteristics will be recorded.

Participants who have successfully responded to ECT in phase I and meet inclusion criteria to continue to the randomised controlled pilot trial in Phase II will be randomised (1:1) to receive four once-weekly infusions of either ketamine or midazolam. The regimen of four weekly infusions was chosen to facilitate subjects travelling for appointments and because ketamine has proven effects as a rapid-acting antidepressant but has not yet been studied as a series of infusions for relapse prevention. Patients and raters will be blind to treatment. The first infusion will be administered within 2 weeks of completing ECT and may be administered as an inpatient or outpatient; further infusions will take place as an outpatient. Each infusion will take $40 \mathrm{~min}$, and monitoring will take place for $200 \mathrm{~min}$ postcommencement of infusion. Ketamine hydrochloride $10 \mathrm{mg} / \mathrm{ml}$ infusion at $0.5 \mathrm{mg} / \mathrm{kg}$ (Pfizer Healthcare Ireland) or midazolam hydrochloride (Hypnovel) $10 \mathrm{mg} /$ $5 \mathrm{ml}$ solution at $0.045 \mathrm{mg} / \mathrm{kg}$ (Roche Products Ireland Ltd) will be made up as $50 \mathrm{ml}$ colourless saline solutions and administered intravenously via an infusion pump. This dose and administration was chosen based on a previous randomised controlled trial of ketamine and midazolam in which these dosages were well-tolerated [51].

During each treatment session, participants will be monitored for heart rate, blood pressure, pulse oximetry and electrocardiogram changes. Adverse or psychotomimetic effects of either agent will be monitored using the CADSS, BPRS (four-item positive symptom subscale), YMRS (mood item) and the PRISE administered before, during and after infusions. Cognitive outcomes will be repeated in week 4 . All other assessment measures will be repeated weekly, including treatment review, HRSD-24 and QIDS-SR.

Participants will be advised not to drive or operate heavy machinery for $24 \mathrm{~h}$ post-commencement of infusions and be provided with information on recent changes to the Road Traffic Act (Ireland) 2014, which includes provisions for roadside intoxication testing. Participants will be asked to ensure they have a nominated adult who can stay with them for $24 \mathrm{~h}$ on outpatient treatment days and will be contacted by a researcher $24 \mathrm{~h}$ after each session to enquire about side effects.

Treatment-as-usual will continue during the trial. Participants will continue to receive pharmacotherapy, psychotherapy or other therapeutic inputs as recommended by their treating team for the duration of the trial. There are no provisions for post-trial care or improving adherence due to the pilot trial design. Premature termination of the trial may take place in the event of new information regarding safety of investigative medicinal products becoming available, unsatisfactory progression of the trial, major breach of data confidentiality or if in the participants' best interests. Subjects have the right to voluntarily discontinue study treatment or withdraw from the study at any time for any reason without any consequences. Subjects must discontinue the investigational medicinal product(s) and be withdrawn from the study for any of the following reasons:

- Withdrawal of consent by the subject

- Any medical condition that the investigator or sponsor determines may jeopardize the subject's safety if she or he continues receiving the study treatment

- Pregnancy

- Ineligibility (either arising during the study or retrospectively having been overlooked at screening) 
- An adverse event which requires discontinuation of the study medication

- Treatment failure and disease progression

- Lack of compliance with the study and/or study procedures (e.g. dosing instructions, study visits)

- Loss to follow-up-at least three documented attempts must be made to contact any subject lost to follow-up.

\section{Outcomes}

The focus of this study is on trial process with assessment of the primary clinical outcome being secondary. However, efficacy data will be collected in the course of the trial and will be reported as part of the study findings.

Process outcomes that will help to inform a future definitive ketamine relapse prevention trial include information on the following:

1. Recruitment methods and rate

2. Willingness of participants to be randomised

3. Willingness of participants to complete assessments

4. Randomisation

5. success of blinding of participants and raters

6. Ability to administer a course of four weekly ketamine infusions

7. Medical safety and acceptability of ketamine infusions in an ECT responder population

8. Rates of adverse dissociative and psychiatric events

9. Adherence to allocated treatment

10. Adherence to follow-up
11. Reasons for drop-out from treatment

12. Reasons for drop-out from follow-up

13. A $95 \%$ confidence interval for the difference between the ketamine and midazolam groups in 6-month relapse rates to help inform a future definitive trial

The primary outcome relating to efficacy (the assessment of which is not a primary objective) is the relapse rate at 6 months as measured by HRSD-24. Subjective mood rating as measured by scores on QIDS-SR is a secondary efficacy outcome. The following safety evaluations will be performed during the study: adverse event monitoring, vital signs, cognitive and clinical assessments. Safety endpoints are:

(i) Tolerability of ketamine vs. midazolam in terms of cognitive outcomes

(ii) Tolerability of ketamine vs. midazolam in terms of psychotomimetic effects as measured by scores on CADSS, BPRS, YMRS and PRISE

(iii) Number of adverse effects in ketamine vs. midazolam groups

This trial has been designed and will be reported in line with the Consolidated Standards of Reporting Trials (CONSORT) guidelines [52] (Fig. 1).

\section{Allocation sequence generation and concealment}

ECT responders will be randomised after post-ECT assessment. Subjects will be randomly assigned to one of

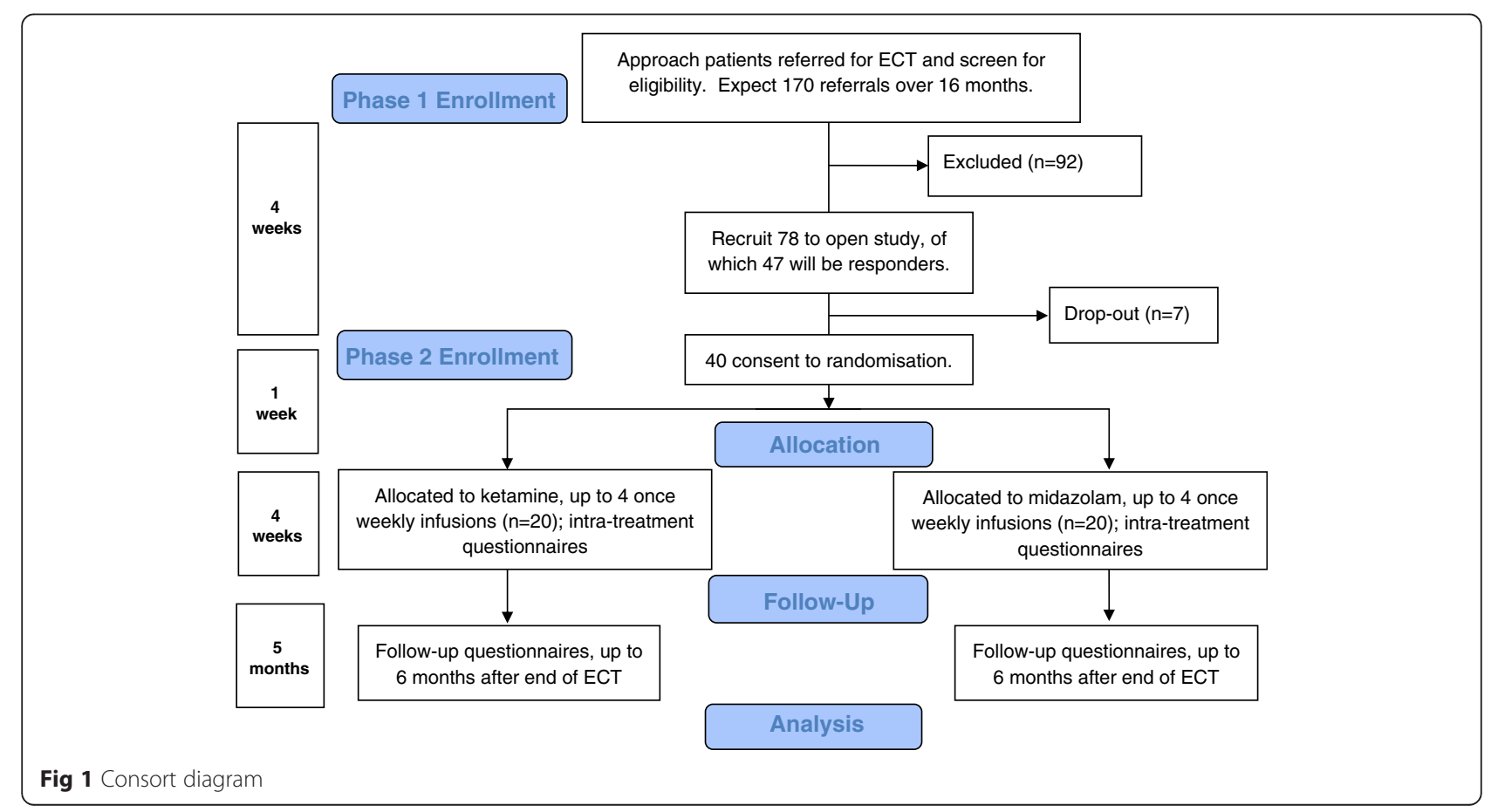


two treatment groups in a 1:1 ratio. Computerised random allocation, using randomly permuted blocks, will be done independently by the Centre for Support and Training in Analysis and Research (CSTAR, University College Dublin, www.cstar.ie). Allocation information will be provided by means of a randomisation list prepared by CSTAR, available only to the anaesthetist to ensure allocation concealment. Researchers involved in ratings will not have access to information regarding treatment allocation.

\section{Blinding and unblinding}

To ensure patient safety during infusions and in the post-infusion period, the anaesthetist administering the ketamine/midazolam infusions will not be blinded but he will not be involved in assessments or data analysis. Patients, raters and the trial statistician will remain blinded. Success of blinding for patients and raters will be assessed after the first treatment. A set of envelopes containing allocation information will remain unopened but may be used where emergency unblinding is indicated. Unblinding for one or all participants will take place if it is in the best interests of the participants.

\section{Statistical methods}

Pilot trial data will be analysed on an intention-to-treat basis for all participants who complete at least one infusion and one post-infusion evaluation. Data analyses will be performed blinded to allocation by the trial statistician. As this is a pilot trial and small numbers of participants are involved, no missing values will be imputed. Data will be analysed using IBM PASW (SPSS) version 22 and "R" (R Foundation for Statistical Computing, Austria).

Demographic and baseline data will be summarised for each treatment group by presenting descriptive statistics. Descriptive statistics will also be used to report: rates of recruitment, willingness to be randomised, willingness to complete assessments, medical/cognitive/psychotomimetic/general adverse events between groups, adherence to allocated treatments, adherence to follow-up between groups and reasons for drop-outs between groups.

Relapse-free survival times will be compared between groups using Kaplan-Meier survival curves and logrank test. As this is a pilot trial and insufficiently powered to achieve statistical significance, there will be no formal comparison of the two treatment groups in the pilot trial. However, Cox proportional hazard regression analysis will provide a $95 \%$ confidence interval for an unadjusted hazard ratio for a future definitive trial.

As this is a pilot trial and small numbers of participants are involved, no missing values will be imputed. As per the pilot trial design, the primary outcomes are those relating to reasons for dropout and these will be presented using descriptive statistics.

\section{Data management}

A trial-specific operating procedure for data quality assurance will be followed by all researchers and involves eight levels of quality assurance. Researchers will be trained in administration of the primary assessment tool used in this study, the HRSD-24, and training will be repeated every 6 months to ensure good inter-rater reliability. The study will comply with the Data Protection (Amendment) Act, 2003, Ireland. All documents will be stored safely in a designated locked filing cabinet in a locked office within the Research Building at St. Patrick's University Hospital, and confidentiality will be observed at all times. With the exception of the informed consent form, subjects will be referred to only by their subject identification number on all study-specific documents, whether hard copies or electronic. Data analysis will take place in another facility (CSTAR, University College Dublin), and data will be anonymised prior to secure transfer to CSTAR for analysis.

\section{Trial management}

The Trial Steering Committee, chaired by an independent clinician and including a service user representative, will meet on a six-monthly basis. The Trial Management Group, comprising trial researchers, will meet on a weekly basis. A Data Monitoring Committee (DMC), chaired by an independent clinical researcher and comprising an independent biostatistician and independent trial methodologist, will meet every 6 months to review blinded data and reports prepared by the trial statistician. The trial results will be published and communicated to participants, and authorship eligibility guidelines of the International Committee of Medical Journal Editors (ICJME) will be followed. There is no intended use of professional authors. Important amendments to the trial protocol will be communicated to all relevant agencies. The sponsor owns the final dataset; access will be managed through the Principal Investigator. There are no contractual agreements limiting access to data for investigators. There are no plans to grant public access to the participant-level dataset or statistical code. Trial processes will not be independently audited due to the pilot trial design.

\section{Discussion}

This is the first registered trial investigating the potential use of ketamine for relapse prevention in depression and the first study to investigate intravenous ketamine for relapse prevention following successful response to ECT. Only one other registered study proposes to investigate this issue; however, that study uses intranasal esketamine (NCT02493868). Previous studies have shown that ketamine has a rapid effect in acute depression, maintained up to 2 weeks, but repeated infusions have not been studied in a recovered population for the purpose of 
relapse prevention. The focus of this pilot trial is on process outcomes to help inform a future definitive trial. Strengths of the study include its double-blind design and use of independent randomisation. Notwithstanding the potential utility of the trial in assessing the safety and practicability of this treatment protocol, there are some limitations. The small number of proposed participants in this pilot trial (up to $n=40$ ) limits the statistical analyses which can be confidently applied to the ones described here. The nationwide catchment area of the trial site may pose difficulties in retention of participants from phase I to phase II due to travel challenges. Treatment-asusual will continue for all participants during the pilot trial, resulting in a heterogeneous participant group. This pragmatic design will, however, improve generalisability of the results and applicability to a future definitive trial.

\section{Trial status}

Recruitment commenced

\section{Additional files}

Additional file 1: Informed consent materials. (DOCX $26 \mathrm{~kb}$ )

Additional file 2: Details of biological studies. (DOCX $25 \mathrm{~kb}$ )

\begin{abstract}
Abbreviations
Version III; BPRS, Brief Psychiatric Rating Scale; CADSS, Clinician-Administered Dissociative States Scale; CSTAR, Centre for Support and Training in Analysis and Research, University College Dublin; CTQ, Childhood Trauma Questionnaire; DSM-IV, Diagnostic and Statistical Manual of Mental Disorders, Fourth Edition; ECT, electroconvulsive therapy; EHQ, Edinburgh Handedness Questionnaire; HRSD-24, Hamilton Depression Rating Scale, 24-item version; K-AMI, Kopelman Autobiographical Memory Interview; MDD, major depressive disorder; MSTRD, Maudsley Staging for Treatment-Resistant Depression; NART, National Adult Reading Test; NMDA, N-methyl-D-aspartate; PRISE, Patient-Rated Inventory of Side Effects; QIDS-SR, Quick Inventory of Depressive Symptoms, Self-Report, 16-item; SCID, Structured Clinical Interview for DSM-IV Axis I Disorders; SMMSE, Standardised Mini Mental State Exam; YMRS, Young Mania Rating Scale
\end{abstract}

\section{Acknowledgements}

This study is supported by a grant from the Health Research Board, Ireland (HRA-POR-2014-604). The funding body has no role in design, collection, analysis and interpretation of data or the preparation of this manuscript.

\section{Funding}

Health Research Award 2014, Health Research Board, Ireland (HRA-POR-2014-604).

\section{Ethics approval and consent to participate}

Approval for this pilot trial was obtained from the joint authorised Research Ethics Committee of St. James' and Tallaght Hospitals, Dublin. Site approval was also obtained from the relevant committee at St. Patrick's University Hospital. Authorisation for the clinical trial was obtained from the Health Products Regulatory Authority of Ireland, the relevant body under the European framework for clinical trials, EudraCT (2014-000339-18). The study will be conducted in accordance with the principles that have their origin in the Declaration of Helsinki [32], in accordance with Good Clinical Practice (GCP), as defined by the International Conference on Harmonisation [33] $(\mathrm{ICH})$, and in accordance with the ethical principles underlying European Union Directive 2001/20/EC and 2005/28/EC. The trial has been registered at clinicaltrials.gov (NCT02414932).

Written informed consent will be obtained by members of the research team using the study-specific consent form (Additional file 1). Potential participants will be provided with an information leaflet and letter of invitation (Additional file 1) and verbal information at the first point of contact with a member of the research team.

\section{Composition, roles and responsibilities}

Co-ordinating centre: Single-centre trial at St. Patrick's University Hospital, Dublin

Trial Steering Committee: Composed of an independent Chair with clinical research background, trial statistician, a service user representative and investigators. The role of the TSC will be to provide overall supervision of the trial, including monitoring trial progress and conduct and advising on scientific credibility. The TSC will meet on a six-monthly basis and consider and act, as appropriate, upon the recommendations of the Data Monitoring Committee (DMC), and will ultimately carry the responsibility for deciding whether the trial needs to be stopped on grounds of safety or efficacy. Endpoint Adjudication Committee: None-for the purposes of this pilot trial, the endpoint is reached when planned recruitment is achieved.

\section{Items from the WHO trial registration data set}

Contact for public queries:

thekeepwellstudy@gmail.com

+35312493385

Contact for scientific person and affiliation:

Martha Finnegan, St. Patrick's University Hospital and Trinity College Institute

of Neuroscience

finnegma@tcd.ie

+35312493385

Public Title: KEtamine for dEPression relapse prevention folloWing

ELectroconvuLsive therapy (The KEEP-WELL Trial)

Scientific Title: KEtamine for dEPression relapse prevention folloWing

ELectroconvuLsive therapy: a randomised pilot trial with blood biomarker evaluation (The KEEP-WELL Trial)

Countries of recruitment: Ireland

Health condition or problem studied: Depression

Target sample size: No formal sample size calculation. Recruitment to cease at $n=15-20$ per group

Recruitment status: Recruiting

Date of first enrolment: 1st April 2015

Date and version of protocol: V1.0, 22.2.15

Study type: Interventional pilot clinical trial

- Randomised

- Double-blind

- Parallel, two-arm

- Placebo-controlled

\section{Role of sponsor and funders in study}

Sponsor's role: The Sponsor has ultimate authority over the study design, trial management, data collection and analysis, interpretation of data, writing of report and submission of report. The Sponsor is directly involved in trial management by membership of the Trial Steering Committee.

Funder's role: No role in study design, data collection, trial management, analysis, interpretation of data, writing of report, submission of report.

\section{Trial sponsor}

Name: St. Patrick's Mental Health Services

Contact person: Professor J Lucey

Postal: Office of the Medical Director, St. Patrick's University Hospital, James'

St., Dublin 8

Email: jlucey@stpatsmail.com

Telephone: +353 12493345

Secondary Sponsors: None

\section{Author details}

${ }^{1}$ Department of Psychiatry and Trinity College Institute of Neuroscience, St. Patrick's University Hospital, James' St., Dublin 8, Ireland. ${ }^{2}$ Trinity College Institute of Neuroscience, Trinity College Dublin, College Green, Dublin 2, Ireland. ${ }^{3}$ School of Pharmacy and Pharmaceutical Sciences, Trinity College Dublin, College Green, Dublin 2, Ireland. ${ }^{4}$ Centre for Training and Research in Analysis and Research, University College Dublin, Belfield, Dublin 4, Ireland. 
Received: 6 April 2016 Accepted: 13 July 2016

Published online: 03 August 2016

\section{References}

1. Kessler RC, Berglund P, Demler O, Jin R, Merikangas KR, Walters EE. Lifetime prevalence and age-of-onset distributions of DSM-IV disorders in the National Comorbidity Survey Replication. Arch Gen Psychiatry. 2005;62(6):593-602.

2. Wittchen HU, Jacobi F, Rehm J, Gustavsson A, Svensson M, Jonsson B, Olesen J, Allgulander C, Alonso J, Faravelli C, et al. The size and burden of mental disorders and other disorders of the brain in Europe 2010. Eur Neuropsychopharmacol. 2011;21(9):655-79.

3. Vos T, Flaxman AD, Naghavi M, Lozano R, Michaud C, Ezzati M, Shibuya K, Salomon JA, Abdalla S, Aboyans V, et al. Years lived with disability (YLDs) for 1160 sequelae of 289 diseases and injuries 1990-2010: a systematic analysis for the Global Burden of Disease Study 2010. Lancet. 2012;380(9859):2163-96.

4. Rush AJ, Trivedi MH, Wisniewski SR, Nierenberg AA, Stewart JW, Warden D, Niederehe G, Thase ME, Lavori PW, Lebowitz BD, et al. Acute and longerterm outcomes in depressed outpatients requiring one or several treatment steps: a STAR*D report. Am J Psychiatr. 2006;163(11):1905-17.

5. UKECTReviewGroup. Efficacy and safety of electroconvulsive therapy in depressive disorders: a systematic review and meta-analysis. Lancet. 2003; 361(9360):799-808.

6. Eranti S, Mogg A, Pluck G, Landau S, Purvis R, Brown RG, Howard R, Knapp M, Philpot M, Rabe-Hesketh S, et al. A randomized, controlled trial with 6-month follow-up of repetitive transcranial magnetic stimulation and electroconvulsive therapy for severe depression. Am J Psychiatr. 2007;164(1):73-81.

7. Semkovska MLS, Dunne R, Kolshus E, Kavanagh A, Jelovac A, Noone M, Carton M, Lambe S, McHugh C, McLoughlin D. Bitemporal versus high-dose unilateral twice-weekly electroconvulsive therapy for depression (EFFECTDep): a pragmatic, randomized, non-inferiority trial. Am J Psychiatr. 2016. http://dx.doi.org/10.1176/appi.ajp.2015.15030372.

8. Semkovska M, McLoughlin DM. Objective cognitive performance associated with electroconvulsive therapy for depression: a systematic review and meta-analysis. Biol Psychiatry. 2010;68(6):568-77.

9. Psychiatrists RCo. The ECT handbook, 3rd edition. 2013.

10. Commission MH. The administration of ECT in approved centres: activity report 2012. 2014.

11. Jelovac A, Kolshus E, McLoughlin DM. Relapse following successful electroconvulsive therapy for major depression: a meta-analysis. Neuropsychopharmacology. 2013;38:2467-74.

12. Excellence NIfHaC. Depression in adults: recognition and management. In: CG 90 Research Recommendations. 2009.

13. Geddes JRCS, Davies C, Furukawa TA, Kupfer DJ, Frank E, et al. Relapse prevention with antidepressant drug treatment in depressive disorders: a systematic review. Lancet. 2003;361:653-61.

14. Sackeim HA, et al. Continuation pharmacotherapy in the prevention of relapse following electroconvulsive therapy: a randomized controlled trial. JAMA. 2001;285(10):1299-307.

15. Petrides $\mathrm{G}$, et al. Continuation ECT: relapse prevention in affective disorders. J ECT. 1994;10(3):189-94.

16. McGirr A, Berlim M, Bond D, Fleck M, Yatham L, Lam R. A systematic review and meta-analysis of randomized, double-blind, placebo-controlled trials of ketamine in the rapid treatment of major depressive episodes. Psychol Med. 2015:45(04):693-704.

17. Xu Y, Hackett M, Carter G, Loo C, Gálvez V, Glozier N, Glue P, Lapidus K, McGirr A, Somogyi AA, Mitchell PB, Rodgers A. Effects of Low-Dose and Very Low-Dose Ketamine among Patients with Major Depression: a Systematic Review and Meta-Analysis. Int J Neuropsychopharmacol. 2016;19(4). Print 2016 Apr. doi:10.1093/ijnp/pyv124.

18. Wan LB1, Levitch CF, Perez AM, Brallier JW, losifescu DV, Chang LC, Foulkes A, Mathew SJ, Charney DS, Murrough JW. Ketamine safety and tolerability in clinical trials for treatment-resistant depression. J Clin Psychiatry. 2015;76(3): 247-52. doi: 10.4088/JCP.13m08852.

19. Murrough JW, losifescu DV, Chang LC, Al Jurdi RK, Green CM, Perez AM, Iqbal S, Pillemer S, Foulkes A, Shah A, et al. Antidepressant efficacy of ketamine in treatment-resistant major depression: a two-site randomized controlled trial. Am J Psychiatr. 2013;170(10):1134-42.

20. Romeo B, et al. Meta-analysis of short-and mid-term efficacy of ketamine in unipolar and bipolar depression. Psychiatry Res. 2015; 230(2):682-8
21. Fond G, Loundou A, Rabu C, Macgregor A, Lançon C, Brittner M, MicoulaudFranchi J-A, Richieri R, Courtet P, Abbar M. Ketamine administration in depressive disorders: a systematic review and meta-analysis. Psychopharmacology. 2014;231(18):3663-76.

22. McCloud TL, Caddy C, Jochim J, Rendell JM, Diamond PR, Shuttleworth C, Brett D, Amit BH, McShane R, Hamadi L, Hawton K, Cipriani A. Ketamine and other glutamate receptor modulators for depression in bipolar disorder in adults. Cochrane Database Syst Rev. 2015; 29(9):CD011611. doi:10.1002/ 14651858.CD011611.pub2.

23. Clements J, Nimmo W, Grant I. Bioavailability, pharmacokinetics, and analgesic activity of ketamine in humans. J Pharm Sci. 1982;71(5):539-42.

24. Perry EB Jr1, Cramer JA, Cho HS, Petrakis IL, Karper LP, Genovese A, O'Donnell E, Krystal JH, D'Souza DC; Yale Ketamine Study Group. Psychopharmacology. 2007;192(2):253-60.

25. Caddy C AB, McCloud TL, Rendell JM, Furukawa TA, McShane R, Hawton K, A C. Ketamine and other glutamate receptor modulators for depression in adults (review). Cochrane Lib. 2015(9).

26. Shiroma PR, et al. Neurocognitive performance and serial intravenous subanesthetic ketamine in treatment-resistant depression. Int J Neuropsychopharmacol. 2014;17(11):1805-13.

27. Murrough JWWL-B, lacoviello B, Collins KA, Solon C, Glicksberg B, et al. Neurocognitive effects of ketamine in treatment-resistant major depression: association with antidepressant response. Psychopharmacology. 2014;231:481-8.

28. McGirr A, et al. A systematic review and meta-analysis of randomized controlled trials of adjunctive ketamine in electroconvulsive therapy: efficacy and tolerability. J Psychiatr Res. 2015;62:23-30.

29. aan het Rot M, Collins KA, Murrough JW, Perez AM, Reich DL, Charney DS, Mathew SJ. Safety and efficacy of repeated-dose intravenous ketamine for treatment-resistant depression. Biol Psychiatry. 2010;67(2):139-45.

30. Association AP. Diagnostic and statistical manual-text revision (DSM-IV-TR): American Psychiatric Association; 2000

31. Dunne R, McLoughlin D. Regional Variation in Electroconvulsive Therapy Use. Ir Med J. 2011;104(3):84-7.

32. Association WM. World Medical Association, Declaration of Helsinki: ethical principles for medical research involving human subjects. Bull World Health Organ. 2001;79(4):373.

33. Group ICOHW. ICH harmonised tripartite guideline: guideline for good clinical practice. In: International Conference on Harmonisation of Technical Requirements for Registration of Pharmaceuticals for Human Use: 1996. 1996.

34. Arain M, Campbell MJ, Cooper CL, Lancaster GA. What is a pilot or feasibility study? A review of current practice and editorial policy. BMC Med Res Methodol. 2010;10:67doi: 10.1186/1471-2288-10-67.

35. Cockrell J, Folstein M. Mini-mental state examination (MMSE). Psychopharmacol Bull. 1988;24(4):689.

36. Hamilton M. A rating scale for depression. J Neurol Neurosurg Psychiatry. 1960;23:56-62.

37. Rush AJ, Trivedi MH, Ibrahim HM, Carmody TJ, Arnow B, Klein DN, Markowitz JC, Ninan PT, Kornstein S, Manber R, et al. The 16-item quick inventory of depressive symptomatology (QIDS), clinician rating (QIDS-C), and self-report (QIDS-SR): a psychometric evaluation in patients with chronic major depression. Biol Psychiatry. 2003;54(5):573-83.

38. First MB, Spitzer RL, Gibbon M, Williams JB. Structured Clinical Interview for DSM-IV axis I Disorders. Clinician Version (SCID-CV). Washington, D.C.: American Psychiatric Press, Inc., 1996.

39. Fekadu A, Wooderson SC, Markopoulou K, Cleare AJ. The Maudsley staging method for treatment-resistant depression: prediction of longerterm outcome and persistence of symptoms. J Clin Psychiatry. 2009; 70(7):952-7.

40. Oldfield RC. The assessment and analysis of handedness: the Edinburgh inventory. Neuropsychologia. 1971;9(1):97-113.

41. Nelson HE, National Adult Reading Test (NART): For the Assessment of Premorbid Intelligence in Patients with Dementia: Test Manual, NFERNelson, Windsor, UK.

42. Bremner JD, Krystal JH, Putnam FW, Southwick SM, Marmar C, Charney DS, Mazure CM. Measurement of dissociative states with the clinician-administered dissociative states scale (CADSS). J Trauma Stress. 1998;11(1):125-36.

43. Overall JE, Gorham DR. The brief psychiatric rating scale. Psychol Rep. 1962; 10(3):799-812.

44. Young RC, Biggs JT, Ziegler VE, Meyer DA. A rating scale for mania: reliability, validity and sensitivity. Br J Psychiatry. 1978;133:429-35. 
45. Wisniewski SR, Rush AJ, Balasubramani G, Trivedi MH, Nierenberg AA, Investigators S. Self-rated global measure of the frequency, intensity, and burden of side effects (PRISE). J Psychiatr Pract. 2006;12(2):71-9.

46. Wechsler D, Laicardi C, Orsini A. WAIS-R: Wechsler adult intelligence scale revised: manual: OS; 1997.

47. Tombaugh TN, Kozak J, Rees L. Normative data stratified by age and education for two measures of verbal fluency: FAS and animal naming. Arch Clin Neuropsychol. 1999;14(2):167-77.

48. Hsieh S, Schubert S, Hoon C, Mioshi E, Hodges JR. Validation of the Addenbrooke's cognitive examination III in frontotemporal dementia and Alzheimer's disease. Dement Geriatr Cogn Disord. 2013;36(3-4):242-50.

49. Kopelman MD, Wilson BA, Baddeley AD. The autobiographical memory interview: a new assessment of autobiographical and personal semantic memory in amnesic patients. J Clin Exp Psychol. 1989;11(5):724-44.

50. Hodge B. ECT accreditation service (ECTAS) standards for the administration of ECT. R Coll Psychiatr. 2015. available at http://www.rcpsych.ac.uk/quality/ qualityandaccreditation/ectclinics/ectas/ectasstandards.aspx.

51. Katalinic N, Lai R, Somogyi A, Mitchell PB, Glue P, Loo CK. Ketamine as a new treatment for depression: a review of its efficacy and adverse effects. Aus N Z J Psychiatry. 2013;47(8):710-27.

52. Schulz KF, Altman DG, Moher D. CONSORT 2010 statement: updated guidelines for reporting parallel group randomised trials. BMC medicine. 2010;8(1):1.

\section{Submit your next manuscript to BioMed Central and we will help you at every step:}

- We accept pre-submission inquiries

- Our selector tool helps you to find the most relevant journal

- We provide round the clock customer support

- Convenient online submission

- Thorough peer review

- Inclusion in PubMed and all major indexing services

- Maximum visibility for your research

Submit your manuscript at www.biomedcentral.com/submit

C Biomed Central 